\title{
EarLy Exercise in blunt Chest wall Trauma: a feasibility trial (ELECT Trial)
}

Dr CE Battle: Welsh Centre for Emergency Medicine Research, Emergency Dept, Morriston Hospital, Swansea, UK, SA6 6NL. ceri.battle@wales.nhs.uk (Corresponding author)

Dr CBC O'Neill: Swansea University Medical School, Swansea University, Swansea, UK.

Mrs Hannah Toghill: Physiotherapy Dept, Morriston Hospital, Swansea Bay University Health Board, Swansea, UK

Mr Luke Newey: Physiotherapy Dept, Morriston Hospital, Swansea Bay University Health Board, Swansea, UK

Professor HA Hutchings: Swansea University Medical School, Swansea University, Swansea, UK.

WORD COUNT: 1190 


\section{ABSTRACT}

Background: The aim was to complete a feasibility study that would test the methods of the main trial, that will investigate whether early thoracic and shoulder girdle exercises reduce chronic pain in patients with blunt chest wall trauma, when compared to normal care. Methods: A single centre, parallel, feasibility randomised controlled trial was completed at a University Teaching Hospital in Wales between June and September 2019. Adult patients with blunt chest wall trauma, admitted to hospital for greater than 24 hours, with no concurrent, immediately life-threatening injuries, were included. The intervention was a simple physiotherapy programme comprising thoracic and shoulder girdle exercises. Feasibility outcome measures included: 1) $80 \%$ or more of identified eligible patients were approached for potential recruitment to the trial 2) $30 \%$ or less of approached, eligible patients dissented to participate in the trial; secondary outcomes: 3) follow up data for patient secondary outcomes can be collected for $80 \%$ or more of patients, 4) there should be no greater than $10 \%$ increase in serious adverse events in the intervention group compared to the control group.

Results: A total of 19/19 (100\%) patients were deemed eligible for the trial and were approached for participation, 5/19 (26\%) eligible patients declined to participate in the trial, follow-up data was collected for $n=10 / 14(71 \%)$ patients and there were no serious adverse events reported in either group.

Conclusions: We have demonstrated that a fully powered randomised clinical trial of the ELECT Trial is feasible.

ISRCTN Trial registration number: ISRCTN 16197429. Date registered: 29th May 2019. https://www.isrctn.com/ISRCTN16197429. 


\section{What is already known on this subject?}

Blunt chest trauma is a difficult condition to manage and many patients report chronic pain at three months post-injury.

What this study adds?

This feasibility study has demonstrated that a fully powered randomised clinical trial investigating the effectiveness of an early exercise programme for patients with blunt chest wall trauma is feasible.

\section{BACKGROUND}

Blunt chest wall trauma accounts for over $15 \%$ of all trauma admissions worldwide, with reported mortality ranging between 4 and 60\%.[1] Chronic pain and disability have been reported in $62 \%$ and $57 \%$ of patients at 3 months post injury respectively.[2] Physiotherapy rehabilitation is recommended as part of multi-disciplinary approach to the management of blunt chest trauma, but evidence-based protocols are lacking.[3] Further research is needed into the optimal management of chronic pain and disability. The aim of this trial was to establish the feasibility and acceptability of a future definitive trial, which will determine whether an exercise programme can be used safely and effectively used to reduce chronic pain in blunt chest wall trauma, in clinical practice in the UK.

\section{METHODS}

This trial received ethics approval by the Wales Research Ethics Committee 6 (Ref: 19-WA0144). This study adhered to the CONSORT extension guidelines for pilot and feasibility trials.

\section{Trial design and randomisation}

This was a single-centre, parallel feasibility randomised controlled trial, initiated in a trauma unit in Wales, over a three month period (June to September 2019). Patients were allocated 
to the trial on a 1:1 ratio to control or intervention arms, using "Sealed Envelope", an independent on-line randomisation software company which is available 24 hours per day.[4]

\section{Population}

Patients admitted to the hospital for 24 hours or more, with isolated blunt chest wall trauma (defined as blunt chest wall injury, with or without radiologically confirmed rib fractures), were included in the trial if they were capable of giving consent to participation and aged 18 and over. Exclusion criteria included: patients lacking capacity to provide informed consent, aged under 18 , or presenting with immediately life-threatening injuries or any concurrent injury precluding participation in the intervention (patients with minor injuries not precluding participation, such as a concurrent knee ligament injury, were not excluded)

\section{Sample size}

The trial had a three-month recruitment period. The aim was to recruit 20 patients, the minimum number considered necessary to test data collection processes based on existing recommendations.[5]

\section{Intervention}

Patients were identified by the hospital's physiotherapy-led chest trauma team, who undertook consent and randomisation. Patients allocated to the intervention group, received standard care (where standard care traditionally involves chest physiotherapy techniques such as breathing exercises and early mobilisation), in addition to a programme of thoracic / shoulder girdle exercises (delivered by the physiotherapist who would routinely manage the patient as part of standard care). This programme was continued by the patient, three times per day, for seven days post-assessment. The exercise programme consisted of shoulder active range of movement exercises trunk active side-flexion, rotation, forward flexion and extension range of movement exercises (all within limits of pain). Participants were instructed to complete each exercise five times, per session. (See Additional file 1 for 
exercise programme). Patients were asked to record adherence to the programme, on the back of the exercise programme. The control group received standard care only.

All participants were asked to complete the Euroqol Quality of Life survey (EQ5D-5L)[6] on initial presentation and two more surveys at three months (EQ5D-5L and the Brief Pain Inventory (BPI)).[7] One survey was posted to the patients at three months, followed by a telephone contact if the postal survey was not received by the research team within two weeks.

\section{Criteria for establishing feasibility}

In order to evaluate the feasibility of a full definitive trial, the trial results were assessed against predetermined success outcome criteria using a traffic light system[8], Primary: 1) adherence to the trial protocol by the physiotherapy team and 2) acceptability of the intervention by the patients. Secondary: 1) ability to retrieve follow up data and 2) safety of the intervention measured by number of serious adverse events.

\section{Data analysis}

Analysis was performed on SPSS (Version 23; IBM, Armonk, NY, USA) using the intentionto-treat principle. Analysis was not powered to detect clinically important effects, as this was a feasibility trial. Results are presented as numbers (percentages), means (standard deviations), and medians (interquartile ranges) where non-normally distributed.

\section{Patient and public involvement}

Two patients recovering from recent blunt chest trauma were members of the ELECT Trial Development Group (TDG) that developed the protocol, specifically advising on the content and delivery of the exercise programme, design of patient-facing documents and methods for follow-up. They continued to sit on the Trial Management Group (TMG), attending research meetings and contributing to the overall running of the trial. Moving forward to the 
main trial, the patient representatives will continue to advise on design of patient-facing documents, protocol modification based on this feasibility and on-going analysis of trial results.

\section{RESULTS}

Patients were recruited over the three month period (June to Sept 2019) and there were no difficulties implementing the protocol. A total of 14 patients were recruited (Figure 1). Loss to follow-up was 2 out of 7 (29\%) patients per arm. In the intervention group, patients reported completing a mean of $79 \%$ (range $62 \%-100 \%$ ) of the exercise programme. No issues were reported by patients completing the exercises, in terms of difficulty completing the programme, serious adverse events or increased levels of pain as a result of the exercise.

The intervention and control groups were mostly comparable at baseline, although hospital length of stay appears longer in the intervention group (Table 1).

Table 1. Baseline Characteristics

\begin{tabular}{|l|c|c|c|}
\hline & Total (n=14) & Control (n=7) & Intervention (n=7) \\
\hline Age (years) & $75(70-80)$ & $75(71-86)$ & $72(60-80)$ \\
\hline Male & $10(71 \%)$ & $4(29 \%)$ & $6(43 \%)$ \\
\hline Female & $4(29 \%)$ & $3(21 \%)$ & $1(7 \%)$ \\
\hline Number of rib fractures (median/IQR) & $4(3-7)$ & $4(3-8)$ & $4(3-6)$ \\
\hline Flail chest & $4(29 \%)$ & $2(14 \%)$ & $2(14 \%)$ \\
\hline Injury mechanism: & & & $2(14 \%)$ \\
$\bullet \quad$ Fall <2 metres & $6(43 \%)$ & $4(29 \%)$ & $3(21 \%)$ \\
$\bullet \quad$ Fall >2 metres & $4(29 \%)$ & $1(7 \%)$ & $2(14 \%)$ \\
$\bullet \quad$ Road traffic accident & $4(29 \%)$ & $2(14 \%)$ & $0(0 \%)$ \\
\hline$\quad$ Pulmonary contusion & $1(7 \%)$ & $1(7 \%)$ & $2(14 \%)$ \\
$\bullet \quad$ Paemothorax & $3(21 \%)$ & $1(7 \%)$ & $2(14 \%)$ \\
\hline$\quad$ Intensive Care & $4(29 \%)$ & $2(14 \%)$ & $2(14 \%)$ \\
• High dependency $\quad$ Ward & $2(14 \%)$ & $0(0 \%)$ & $0(\%)$ \\
\hline Intercostal chest drain & $2(14 \%)$ & $2(14 \%)$ & $2(14 \%)$ \\
\hline Highest level of care: & $4(29 \%)$ & $2(14 \%)$ & $5(36 \%)$ \\
\hline Complications & $8(57 \%)$ & $3(21 \%)$ & $2(14 \%)$ \\
\hline
\end{tabular}




\begin{tabular}{|l|c|c|c|}
\multicolumn{1}{|c|}{ - Type 2 respiratory failure } & $1(7 \%)$ & $0(0 \%)$ & $1(7 \%)$ \\
\hline Mechanical ventilation & $0(0 \%)$ & $0(0 \%)$ & $0(0 \%)$ \\
\hline ICU length of stay & $0(0-1)$ & $0(0-1)$ & $0(0-1)$ \\
\hline Total hospital length of stay & $8(4-15)$ & $4(2-10)$ & $10(7-17)$ \\
\hline Discharged home & $14(100 \%)$ & $7(100 \%)$ & $7(100 \%)$ \\
\hline
\end{tabular}

Number (\%), median (IQR)

\section{Feasibility criteria}

All pre-set feasibility criteria achieved a green or amber status (Table 2). A mixture of postal and telephone follow up contacts were required for survey completion at three months.

Table 2: Feasibility outcomes

\begin{tabular}{|l|l|l|}
\hline Feasibility Criteria & Result & $\begin{array}{l}\text { Feasibility } \\
\text { assessment }\end{array}$ \\
\hline Primary outcomes & & \\
\hline $\begin{array}{l}\text { 1) } 80 \% \text { or more of identified eligible patients } \\
\text { were approached for potential recruitment to } \\
\text { the trial }\end{array}$ & $\begin{array}{l}\mathrm{n}=19 / 19(100 \%) \text { patients were } \\
\text { deemed eligible for the trial and } \\
\text { were approached for participation } \\
\mathrm{n}=5 / 19(26 \%) \text { eligible patients } \\
\text { declined to participate in the trial }\end{array}$ & GREEN \\
\hline $\begin{array}{l}\text { 2) } 30 \% \text { or less of approached, eligible patients } \\
\text { dissented to participate in the trial }\end{array}$ & GREN \\
\hline Secondary outcomes & $\begin{array}{l}\text { Follow-up data collected for } \\
n=10 / 14 ~(71 \%) \text { of patients }\end{array}$ & AMBER \\
\hline $\begin{array}{l}\text { 3) Follow up data for patient secondary } \\
\text { outcomes can be collected for } 80 \% \text { or more of } \\
\text { patients }\end{array}$ & $\begin{array}{l}\text { There were no serious adverse } \\
\text { events reported in either group } \\
\text { 4) There should be no greater than } 10 \% \\
\text { increase in serious adverse events in the } \\
\text { intervention group compared to the control } \\
\text { group }\end{array}$ & GREEN \\
\hline
\end{tabular}

number of cases (percent). Feasibility criteria traffic light system: GREEN: feasibility criteria achieved, AMBER: feasibility criteria not achieved, but progression is possible with some minor protocol modifications, RED:

Feasibility criteria not achieved and progression to a full trial is not possible

\section{DISCUSSION}

Although pre-set feasibility criteria were met, this feasibility trial has highlighted some additional minor modifications (not included in original feasibility criteria) that need to be made to the methods prior to moving forward to the full trial. Specifically, the recruitment rate was lower than expected as the trial ran over a three month period in the summer, however this data will inform the length of time needed for recruitment in the main trial. We will also 
need to consider strategies for ensuring optimal recruitment of all potential patients and minimising attrition.

There were a number of limitations in this feasibility study. We did not include adherence to the programme as a progression criteria. This could have been a potential variable precluding progression to full trial, however the reported adherence (mean of $79 \%$ of exercise programme completed) suggested that progression to full trial is feasible. The lower than planned recruitment rate was also a limitation of the trial, however we will be able to conduct a more accurate sample size, with this knowledge, moving forward to the full trial. A qualitative analysis was not completed due to limited funding, but will be included in the full trial. In addition, we will also need to consider the fidelity of intervention delivery, once more sites and physiotherapists are involved.

In conclusion, this work has demonstrated that with some minor modifications, progression to the full definitive impact trial is feasible.

Acknowledgements: The authors acknowledge the contribution of work of the patient representatives Mrs Hazel Spencer and Ms Joanne Prosser.

Contributors: All authors contributed to the conception and design of the trial. $\mathrm{CB}, \mathrm{HH}$ and $\mathrm{LN}$ managed the running of the trial. $\mathrm{CB}, \mathrm{HH}$ and $\mathrm{CO} N \mathrm{~N}$ completed the data analysis. $\mathrm{CB}$, $\mathrm{HH}, \mathrm{HT}$, LN and CO'N wrote the initial draft and all authors contributed to its revision.

Funding: This trial was supported by a Pathway to Portfolio Grant from Health and Care Research Wales.

Disclaimer: The funding sources have no role in the design of this trial. The views expressed are those of the author(s) and not necessarily those of the NHS, Health and Care Research Wales, the NIHR or the Department of Health.

Competing interests: There are no competing interests to declare

Ethics approval: Wales Research Ethics Committee 6 (16/WA/0144) 
Provenance and peer review: Not commissioned; externally peer reviewed

Data sharing statement: All trial investigators will maintain full autonomy and involvement in the design, conduct and reporting of the trial, with all having full access to the data

\section{REFERENCES}

1) Battle CE, James $K$, Hutchings $H$, et al. Risk factors for the development of complications in blunt chest wall trauma: a retrospective study. Injury. 2013;44:1171-1176.

2) Carrie C, Guemmar Y, Cottenceau V, de Molliens L, Petit L, Sztark F, et al. Long-term disability after blunt chest trauma: don't miss chronic neuropathic pain. Injury. 2019;113-118.

3) Unsworth A, Curtis K, Asha SE. Treatments for blunt chest trauma and their impact on patient outcomes and health service delivery. Scand J Trauma Resusc Emerg Med. 2015;23:17.

4) Sealed Envelope Ltd. 2019. https://www.sealedenvelope.com/simple-randomiser/v1/ . Accessed on $21^{\text {st }}$ Jan 2019

5) Lancaster GA, Dodd SR, Williamson PR. Design and analysis of pilot studies: recommendations for good practice. J Eval Clin Pract. 2004;10:307-12.

6) The EuroQol Group. EuroQol - a new facility for the measurement of health-related quality of life. Health Policy. 1990;16:199-208.

7) Cleeland CS. The Brief Pain Inventory: user guide. 2009. https://www.mdanderson.org/documents/Departments-and-Divisions/SymptomResearch/BPI UserGuide.pdf. Accessed 21 ${ }^{\text {st }}$ Jan 2019. 
8) Battle C, Hutchings HA, Driscoll T, O'Neill C, Groves S, Watkins A, et al. A multicentre randomised feasibility STUdy evaluating the impact of a prognostic model for Management of BLunt chest wall trauma patients: STUMBL Trial. BMJ Open 2019;9:e029187

FIGURE TITLES / LEGENDS

Figure 1: STUMBL CONSORT diagram

ADDITIONAL FILES

Additional file 1: ELECT Trial Exercise Programme

Exercise programme used as the intervention in ELECT trial 